\title{
Echinoderm Fauna of Kosrae, The Federation States of Micronesia
}

\author{
Taekjun Lee ${ }^{1}$, Sook Shin ${ }^{2, *}$ \\ ${ }^{1}$ College of Life Sciences and Biotechnology, Korea University, Seoul 136-701, Korea \\ ${ }^{2}$ Department of Life Science, Sahmyook University, Seoul 139-742, Korea
}

\begin{abstract}
Micronesia is an island nation located north of Papua New Guinea in the Pacific Ocean. A survey was executed on Kosrae, which is one of the four main islands of Micronesia, during January 23-30, 2011 and January $8-15,2012$. The echinoderms were collected at eight intertidal areas and at 12 SCUBA diving points at depths of $10-50 \mathrm{~m}$ in the subtidal zone. They were identified based on morphological characteristics. In total, 50 species from 276 individuals were identified: three crinoids, 11 asteroids, seven ophiuroids, 10 echinoids, and 19 holothuroids. Among them, two crinoids, five asteroids, three ophiuroids, four echinoids and two holothuroids were newly recorded from Kosrae.
\end{abstract}

Keywords: taxonomy, Echinodermata, fauna, Kosrae, Micronesia

\section{INTRODUCTION}

Kosrae is one of four states in the Federated States of Micronesia (FSM) that stretch 1,800 miles across the North Pacific Ocean and comprise 607 small islands. Kosrae is called "the sleeping lady" because of it shape. It is one of the most picturesque mountains located about $5^{\circ}$ north of the equator and is the most eastern island in the FSM (Edward, 2002). The coral reefs surrounding the island are kept in pristine condition through an extensive mooring buoy system that was installed and is maintained by concerned expat dive operators with the help of the government Marine Resources office. Specific information on the echinoderm fauna of Micronesia is included in the reports of Kerr (1994) and Kerr et al. (2007, 2008). The Yap of Micronesia was surveyed by Kerr et al. (2007), and a total of 66, including 16 unidentified species were collected from 19 different sites five crinoids (four unidentified), 14 asteroids (two unidentified), 14 echinoids (three unidentified), and 33 holothuroids (seven unidentified). Kerr (1994) and Kerr et al. (2007) investigated the echinoderm fauna of Kosrae. Kerr (1994) reported 28 holothuroids collected at nine sites, and Kerr et al. (2007) reported 74 species collected at 21 sites, which included 20 unidentified species: four crinoids (three unidentified), 11 asteroids (two unidentified), 11 ophiuroids (seven unidentified), eight echinoids (three unidentified), and 40 holothuroids (five unidentified). We surveyed eight intertidal sites and 12 SCUBA diving points around Kosrae for echinoderm fauna and assembled a checklist of echinoderms for this island.

\section{MATERIALS AND METHODS}

We collected samples at 20 Kosrae sites during January 2428, 2011 and January 9-13, 2012 (Fig. 1), and the GPS coordinates of each site were recorded (Table 1). Most intertidal zones were coral sand and rocky regions, and six subtidal zones were investigated by SCUBA diving. All collected specimens were preserved in $95 \%$ ethyl alcohol and were identified based on their morphological characters. The important morphological characters were photographed by digital camera, stereo- and light-microscopes (Nikon D7000, Nikon SMZ1000, Nikon Eclipse 80i; Nikon Co., Tokyo, Japan), and the specimens were identified based mainly on Clark and Rowe (1971) and Rowe and Doty (1977).

\section{RESULTS}

The specimens collected for the Kosrae faunistic study were (c) This is an Open Access article distributed under the terms of the Creative Commons Attribution Non-Commercial License (http://creativecommons.org/ licenses/by-nc/3.0/) which permits unrestricted non-commercial use, distribution, and reproduction in any medium, provided the original work is properly cited.

pISSN 2234-6953 eISSN 2234-8190
*To whom correspondence should be addressed

Tel: 82-2-3399-1717, Fax: 82-2-3399-1729

E-mail: shins@syu.ac.kr 


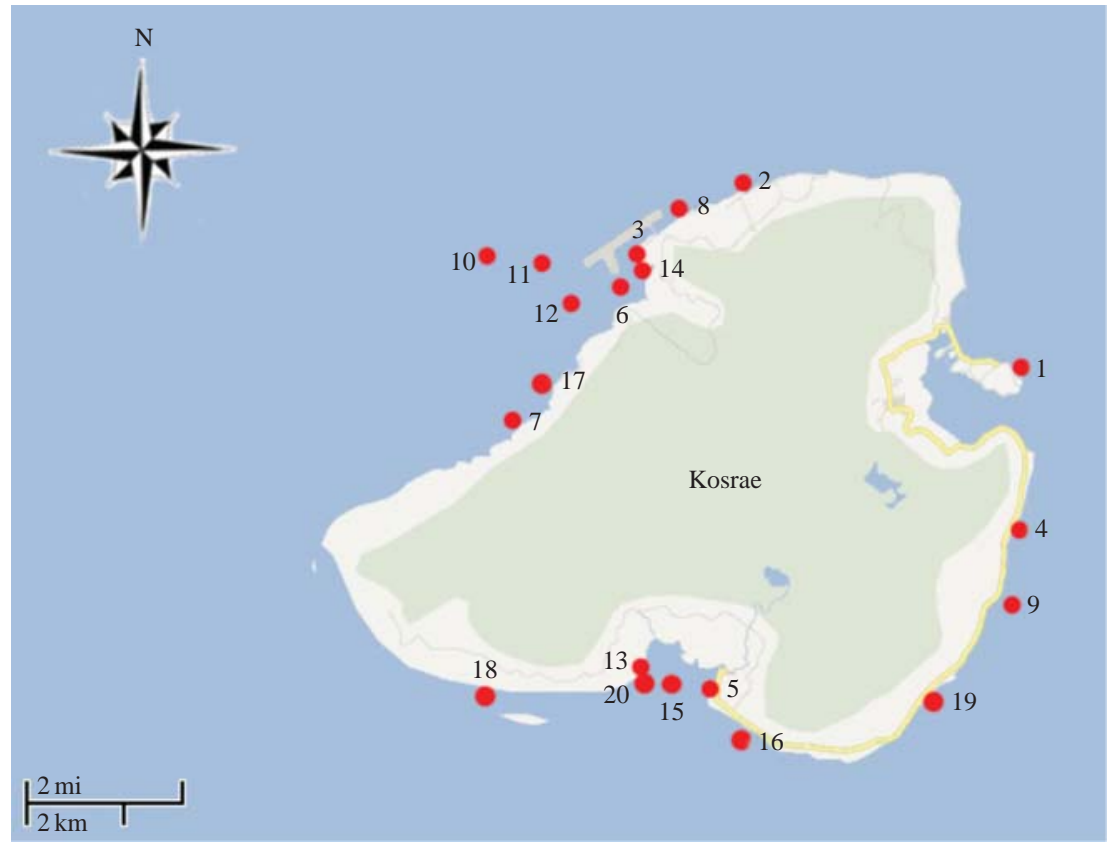

Fig. 1. A map showing the Kosrae collection sites.

Table 1. Geological information for the collection sites around Kosrae

\begin{tabular}{clrc}
\hline Station & Collection site & Date & GPS \\
\hline 1 & Lelu intertidal & 25 Jan 2011 & $5^{\circ} 19^{\prime} 58^{\prime \prime} \mathrm{N}, 163^{\circ} 01^{\prime} 54^{\prime \prime} \mathrm{E}$ \\
2 & Tafunsak intertidal 1 & 26 Jan 2011 & $5^{\circ} 21^{\prime} 55^{\prime \prime} \mathrm{N}, 162^{\circ} 58^{\prime} 42^{\prime \prime} \mathrm{E}$ \\
3 & Tafunsak intertidal 2 & 9 Jan 2012 & $5^{\circ} 21^{\prime} 17^{\prime \prime} \mathrm{N}, 162^{\circ} 57^{\prime} 41^{\prime \prime} \mathrm{E}$ \\
4 & Malem intertidal & 10 Jan 2012 & $5^{\circ} 18^{\prime} 04^{\prime \prime} \mathrm{N}, 163^{\circ} 01^{\prime} 53^{\prime \prime} \mathrm{E}$ \\
5 & Utwa intertidal & 11 Jan 2012 & $5^{\circ} 16^{\prime} 18^{\prime \prime} \mathrm{N}, 162^{\circ} 58^{\prime} 28^{\prime \prime} \mathrm{E}$ \\
6 & Tafunsak intertidal 3 & 11 Jan 2012 & $5^{\circ} 21^{\prime} 02^{\prime \prime} \mathrm{N}, 162^{\circ} 57^{\prime} 37^{\prime \prime} \mathrm{E}$ \\
7 & Walung intertidal & 12 Jan 2012 & $5^{\circ} 19^{\prime} 24^{\prime \prime} \mathrm{N}, 162^{\circ} 56^{\prime} 08^{\prime \prime} \mathrm{E}$ \\
8 & Tafunsak intertidal 4 & 13 Jan 2012 & $5^{\circ} 21^{\prime} 44^{\prime \prime} \mathrm{N}, 162^{\circ} 58^{\prime} 01^{\prime \prime} \mathrm{E}$ \\
9 & Malem diving 1 & $5^{\circ} 17^{\prime} 16^{\prime \prime} \mathrm{N}, 163^{\circ} 01^{\prime} 50^{\prime \prime} \mathrm{E}$ \\
10 & Tafunsak diving 1 & 24 Jan 2011 & $5^{\circ} 21^{\prime} 23^{\prime \prime} \mathrm{N}, 162^{\circ} 55^{\prime} 48^{\prime \prime} \mathrm{E}$ \\
11 & Tafunsak diving 2 & $5^{\circ} 21^{\prime} 10^{\prime \prime} \mathrm{N}, 162^{\circ} 56^{\prime} 50^{\prime \prime} \mathrm{E}$ \\
12 & Tafunsak diving 3 & $5^{\circ} 19^{\prime} 44^{\prime \prime} \mathrm{N}, 162^{\circ} 56^{\prime} 32^{\prime \prime} \mathrm{E}$ \\
13 & Utwa diving 1 & $5^{\circ} 16^{\prime} 25^{\prime \prime} \mathrm{N}, 162^{\circ} 57^{\prime} 35^{\prime \prime} \mathrm{E}$ \\
14 & Tafunsak diving 4 & 26 Jan 2011 & $5^{\circ} 21^{\prime} 01^{\prime \prime} \mathrm{N}, 162^{\circ} 57^{\prime} 20^{\prime \prime} \mathrm{E}$ \\
15 & Utwa diving 2 2011 & $5^{\circ} 16^{\prime} 13^{\prime \prime} \mathrm{N}, 162^{\circ} 58^{\prime} 09^{\prime \prime} \mathrm{E}$ \\
16 & Utwa diving 3 & 27 Jan 2011 & $5^{\circ} 15^{\prime} 46^{\prime \prime} \mathrm{N}, 162^{\circ} 59^{\prime} 01^{\prime \prime} \mathrm{E}$ \\
17 & Walung diving & 28 Jan 2011 & $5^{\circ} 19^{\prime} 46^{\prime \prime} \mathrm{N}, 162^{\circ} 56^{\prime} 36^{\prime \prime} \mathrm{E}$ \\
18 & Utwa diving 4 & 10 Jan 2012 & $5^{\circ} 15^{\prime} 48^{\prime \prime} \mathrm{N}, 162^{\circ} 55^{\prime} 46^{\prime \prime} \mathrm{E}$ \\
19 & Malem diving 2 & 11 Jan 2012 & $5^{\circ} 16^{\prime} 08^{\prime \prime} \mathrm{N}, 163^{\circ} 01^{\prime} 01^{\prime \prime} \mathrm{E}$ \\
20 & Utwa diving 5 & 11 Jan 2012 & $5^{\circ} 16^{\prime} 19^{\prime \prime} \mathrm{N}, 162^{\circ} 57^{\prime} 37^{\prime \prime} \mathrm{E}$ \\
\hline
\end{tabular}

identified as 62 species in 50 genera: three crinoids (sea lily), 11 asteroids (sea star), seven ophiuroids (brittle star), nine echinoids (sea urchin), and 20 holothuroids (sea cucumber). Of these, 16 echinoderms including two crinoids (Oxycomanthus bennetti and Phanogenia gracilis), five asteroids (Archaster typicus, Aquilonastra burtonii, Echinaster luzonicus, Fromia nodosa and Ophidiaster granifer), three ophiuroids
(Ophiactis savignyi, Ophioconis permixta, and Ophiomastix caryophyllata), four echinoids (Diadema savignyi, Echinothrix calamaris, Echinometra mathaei, and Parasalenia gratiosa) and two holothuroids (Labidodemas rugosum and Opheodesoma clarki), which are marked with asterisks (*) in the following list, were newly recorded on Kosrae and were described with brief remarks. 
Phylum Echinodermata Klein, 1734

Subphylum Crinozoa Matsumoto, 1929

Class Crinoidea Müller, 1821

Order Comatulida A.H. Clark, 1908

Family Comasteridae A.H. Clark, 1908

Subfamily Comasterinae A.H. Clark, 1909

Genus Comaster L. Agassiz, 1836

\section{Comaster schlegelii (Carpenter, 1881)}

Material examined. 1 specimen, Malem diving 1, 24 Jan 2011; 2 specimens, Tafunsak diving 2, 26 Jan 2012; 1 specimen, Utwa diving 3, 10 Jan 2012.

Distribution. Micronesia (Kosrae, Yap), southern Japan, southern China, Philippines, Palau, Guam, South Pacific, northern Australia, Indo-West Pacific, Bengal Bay.

Genus Oxycomanthus Rowe, Hoggett, Birtles and Vail, 1986

*2. Oxycomanthus bennetti (Müller, 1841)

Alecto bennetti Müller, 1841: 187.

Cenolia bennetti HL Clark, 1946: 36.

Oxycomanthus bennetti: Rowe and Gates, 1995: 149; Messing, 2012: 246787.

Material examined. 1 specimen, Malem diving 1, 24 Jan 2011; 1 specimen, Utwa diving 3, 10 Jan 2012.

Distribution. Micronesia (Kosrae), southern Japan, southern China, Philippines, Palau, Guam, South Pacific, northern Australia, Indo-West Pacific, Bengal Bay.

Remarks. This species has various body colors (Messing, 2007). Brachials are brown and some terminal parts are white. Pinnules have black basal parts and yellowish tips.

Subfamily Phanogeniinae White and Messing, 2001

Genus Phanogenia Lovén, 1866

\section{*3. Phanogenia gracilis (Hartlaub, 1890)}

Actinometra gracilis Hartlaub, 1890: 170; AH Clark, 1908a: 684; $1912: 38$.

Comaster gracilis AH Clark, 1908a: 686; 1912: 38; AM Clark and Rowe, 1971: 6.

Phanogenia gracilis AH Clark, 1908b: 124; 1909: 392; Messing, 2012: 246804.

Phanogenia minima AH Clark, 1909: 392.

Comaster minima AH Clark, 1912: 89; 1921: 706; Rowe and Gates, 1995: 146.

Comaster minimus AH Clark, 1926: 184.

Material examined. 2 specimens, Malem diving 1, 24 Jan
2011; 1 specimen, Tafunsak diving 1, 25 Jan 2011; 1 specimen, Utwa diving 3, 10 Jan 2012.

Distribution. Micronesia (Kosrae), southern China, Philippines, Palau, Guam, Indo-West Pacific.

Remarks. Messing (2007) described two forms of $P$. gracilis that occurred at Palau: 1) numerous (up to 120) and short (usually $<12 \mathrm{~cm}$ ) arms, 2) fewer (usually $<60$ ) and long (up to $25 \mathrm{~cm}$ ) arms. Our specimens corresponded to the latter type and the body color is brown.

Subphylum Asterozoa von Zittel, 1895

Class Asteroidea de Blainville, 1830

Order Valvatida Perrier, 1884

Family Acanthasteridae Sladen, 1889

Genus Acanthaster Gervais, 1841

\section{Acanthaster planci (Linnaeus, 1758)}

Material examined. 1 specimen, Malem diving 1, 24 Jan 2011.

Distribution. Micronesia (Kosrae, Yap), Guam, Palau, New Zealand (Kermadec Islands), Indo-West Pacific, eastern Africa (Mauritius, Madagascar), Red Sea.

Family Archasteridae Viguier, 1878

Genus Archaster Müller and Troschel, 1840

\section{*5. Archaster typicus Müller and Troschel, 1840}

(Fig. 2A)

Archaster typicus Müller and Troschel, 1840: 104; HL Clark, 1946: 79; AM Clark and Rowe, 1971: 47; Rowe and Gates, 1995: 25; Mah, 2012: 213119.

Material examined. 1 specimen, Tafunsak intertidal 3, 11 Jan 2012.

Distribution. Micronesia (Kosrae), southern Japan, southern China, Philippines, Palau, Guam, West Caroline Islands, South Pacific, Hawaii, northern Australia, Indo-West Pacific, Bengal Bay, West Indian Ocean.

Remarks. This is the most known species of the genus Archaster and is widely distributed in tropical regions of West Pacific and Indo-West Pacific (Clark, 1946; Clark and Rowe, 1971). This specimen was slightly burrowed into the coral sand and was collected in the intertidal zone at night.

Family Asterinidae Gray, 1840

Genus Aquilonastra O'Loughlin, 2004

\section{*6. Aquilonastra burtonii (Gray, 1840)}

(Fig. 3A, D, G-I)

Asterina burtonii Gray, 1840: 289; AM Clark, 1952: 207. 

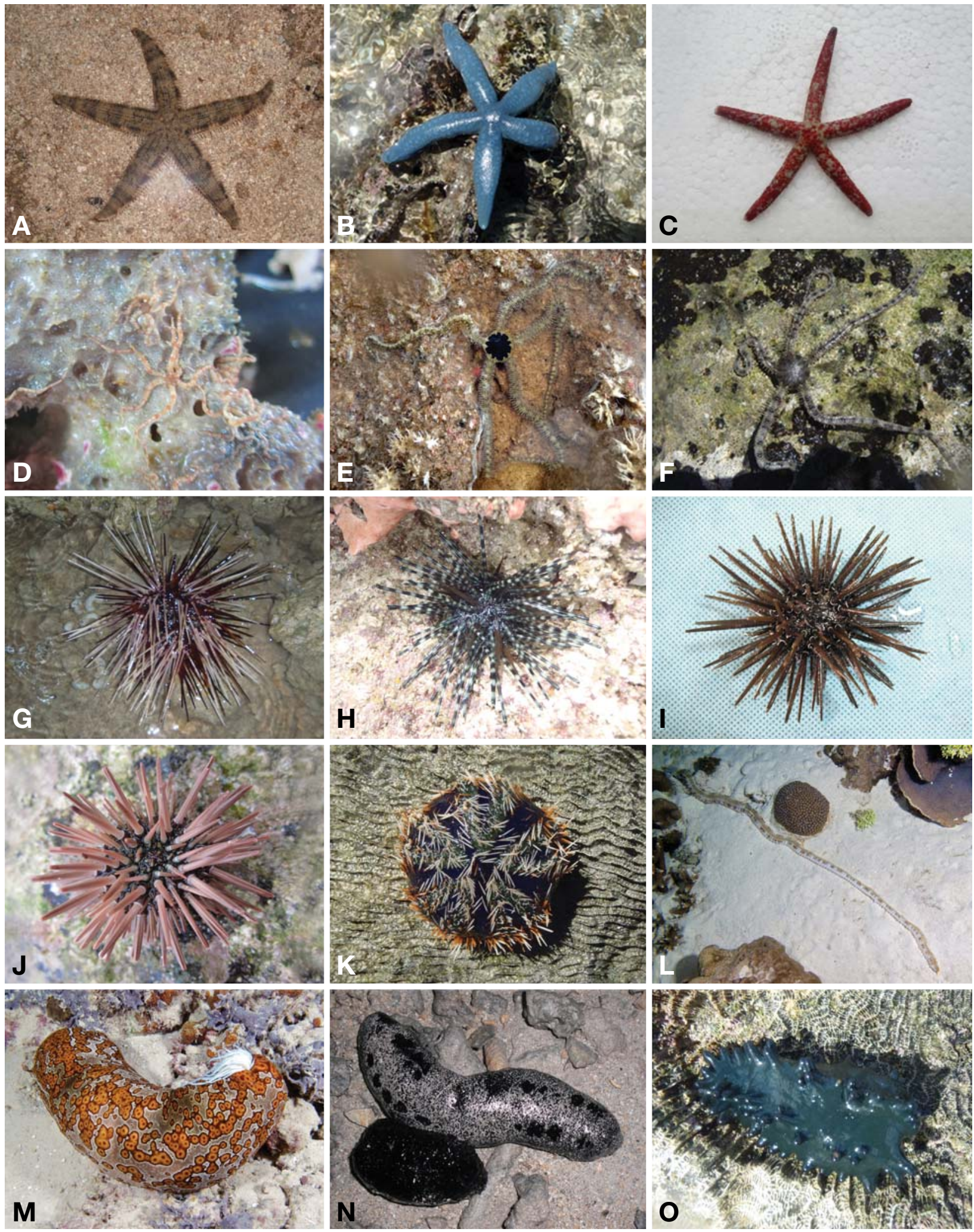

Fig. 2. Some echinoderms collected around Kosrae. A, Archaster typicus; B, Linckia laevigata; C, L. multifora; D, Ophiactis savignyi; E, Ophiocoma erinaceus; F, O. scolopendrina; G, Echinothrix clamaris; H, Echinothrix diadema; I, Parasalenia gratiosa; J, Echinometra mathaei; K, Tripneustes gratilla; L, Euapta godeffroyi; M, Bohadschia argus; N, Holothuria (Halodeima) atra; O, Stichopus chloronotus. 
A

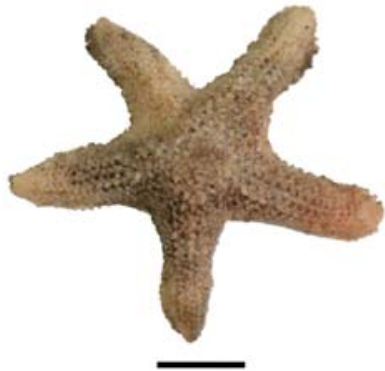

D
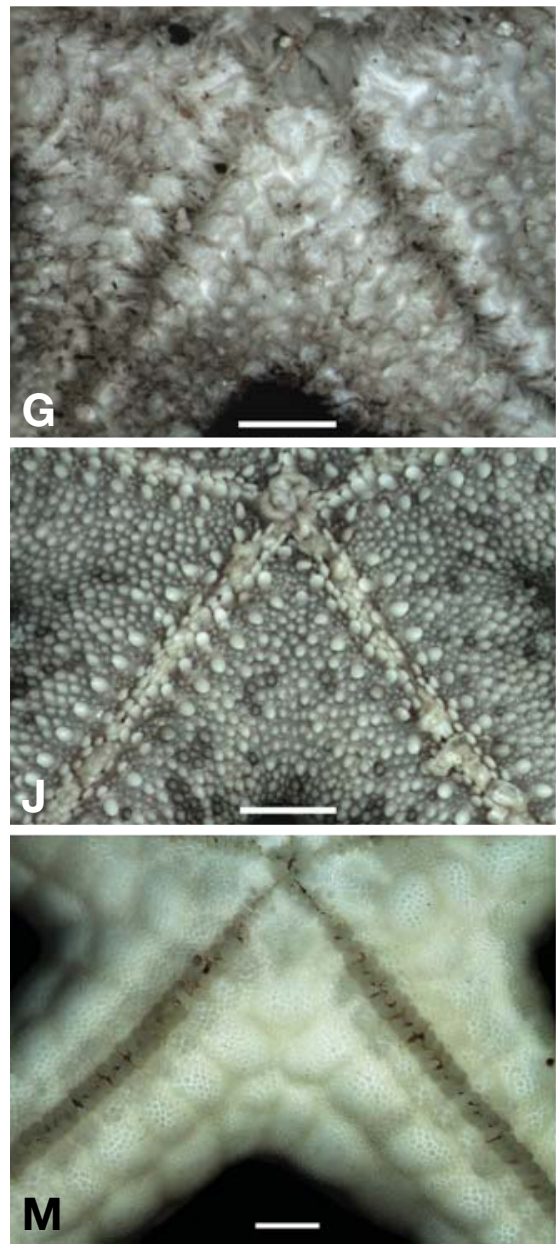

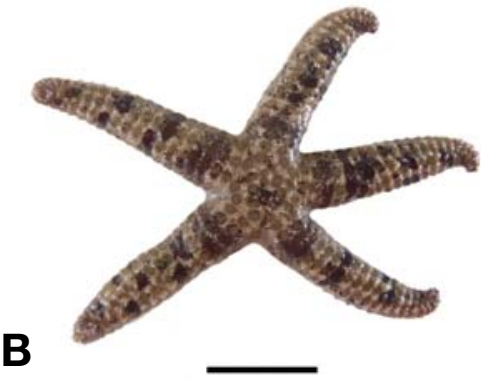

E
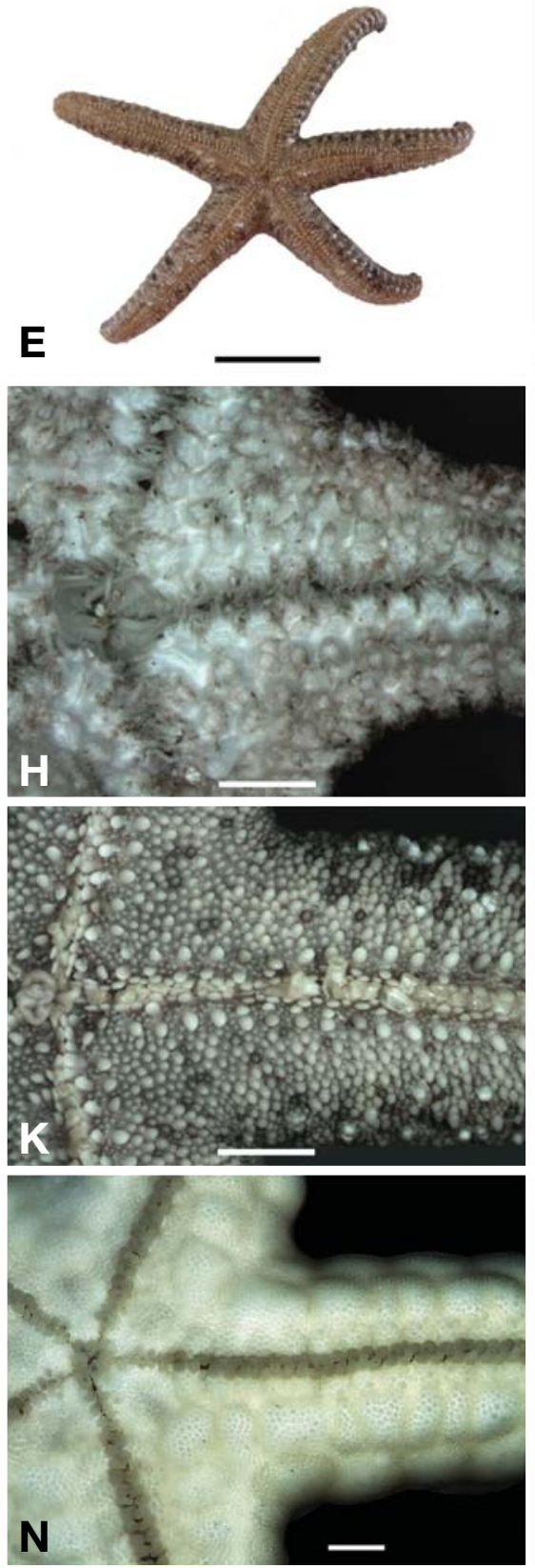

C

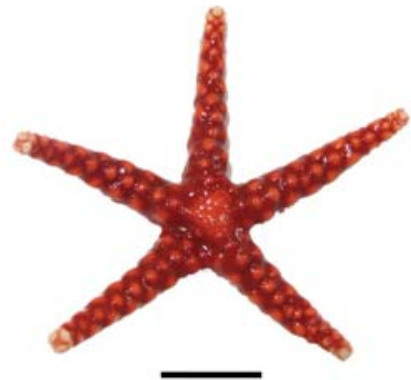

$\mathbf{F}$
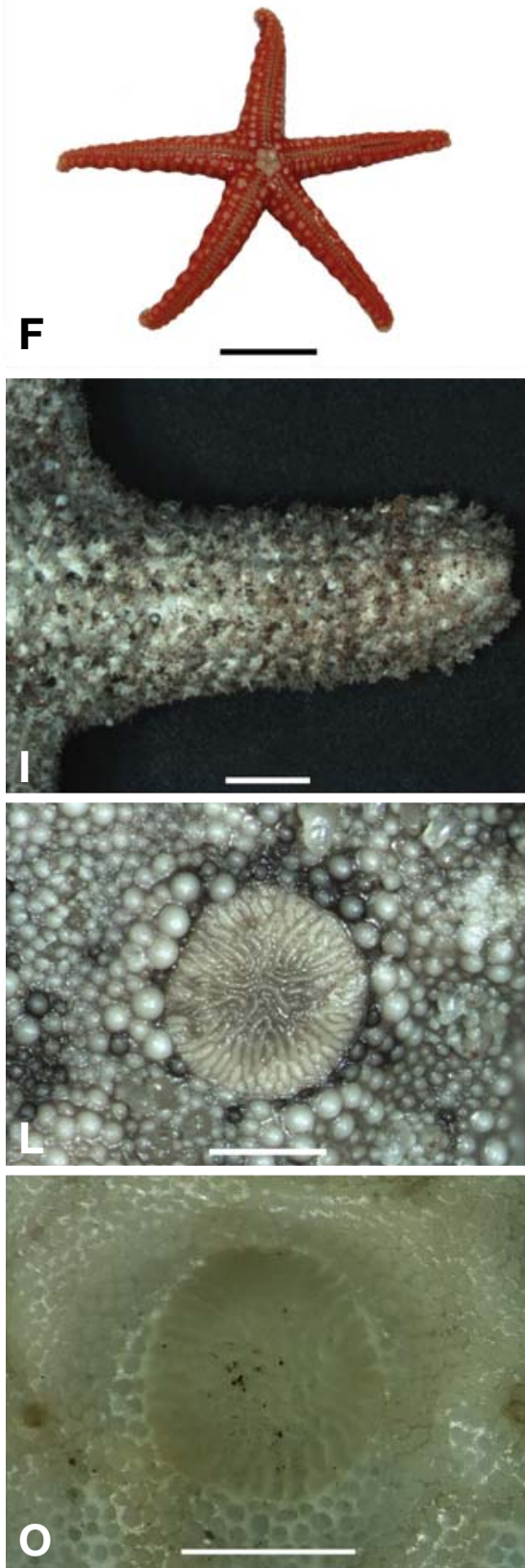

Fig. 3. Aquilonastra burtonii $(A, D, G-I)$, Ophidiaster granifer $(B, E, J-L)$, and Fromia nodosa $(C, F, M-O)$. A-C, Dosal side; D-F, Ventral side; G, H, J, K, M, N, Oral part and ventral side of arm; I, Dorsal side of arm; L, O, Madreporite. Scale bars: $A$, $D=0.5 \mathrm{~cm}$, $B, C, E, F=1 \mathrm{~cm}, G-I, L-O=1 \mathrm{~mm}, J, K=2 \mathrm{~mm}$. 
Asterina burtoni: AM Clark and Rowe, 1971: 68.

Asterina wega Perrier, 1869: 102.

Aquilonastra burtoni O'Loughlin and Waters, 2004: 11; Mah, 2012: 459524.

Aquilonastra burtonii O'Loughlin and Rowe, 2006: 268.

Material examined. 2 specimens, Malem diving 1, 24 Jan 2011.

Distribution. Micronesia (Kosrae), Philippines, South Pacific, Hawaii, northern Australia, Indian Ocean, Arabian Sea, eastern Africa, Red Sea, eastern Mediterranean Sea.

Remarks. Body color is quite diverse: greenish gray, pale gray, brown, mottled brown, orange, red, and mottled red (O'Loughlin and Rowe, 2006). The body color of our specimen is greenish gray.

Family Ophidiasteridae Verrill, 1870

Genus Linckia Nardo, 1834

\section{Linckia guildingi Gray, 1840}

Material examined. 1 specimen, Walung diving, 11 Jan 2012; 1 specimen, Walung intertidal, 12 Jan 2012.

Distribution. Micronesia (Kosrae, Yap), southern China, Guam, Indo-West Pacific, Africa, Caribbean Sea, Central America, Gulf of Mexico.

\section{Linckia laevigata (Linnaeus, 1758) (Fig. 2B)}

Material examined. 12 specimens, Lelu intertidal, 25 Jan 2011; 1 specimen, Tafunsak intertidal 1, 25 Jan 2011; 1 specimen, Tafunsak intertidal 3, 11 Jan 2012; 6 specimens, Walung intertidal, 12 Jan 2012; 1 specimen, Tafunsak intertidal 4, 13 Jan 2012.

Distribution. Micronesia (Kosrae, Yap), Korea (Jeju Island), Japan, southern China, Taiwan, Hong Kong, Guam, Hawaii, Australia, Indo-West Pacific, eastern Africa (Mauritius, Madagascar, Mozambique, Tanzania), Red Sea.

\section{Linckia multifora (Lamarck, 1816) (Fig. 2C)}

Material examined. 1 specimen, Utwa diving 4, 11 Jan 2012; 2 specimens, Malem diving 2, 13 Jan 2012.

Distribution. Micronesia (Kosrae, Yap), southern China, Guam, Australia, Indo-West Pacific, eastern Africa (Madagascar), Red Sea.

Genus Ophidiaster L. Agassiz, 1836

*10. Ophidiaster granifer Lütken, 1871 (Fig. 3B, E, J-L) Ophidiaster granifer Lütken, 1871: 276; HL Clark, 1921: 81;
1946: 121; AM Clark and Rowe, 1971: 60; Rowe and Gates, 1995: 91; Mah, 2012: 212302.

Ophidiaster trychnus Fisher, 1913: 215.

Material examined. 1 specimen, Walung intertidal, 12 Jan 2012.

Distribution. Micronesia (Kosrae), southern Japan, southern China, Philippines, Palau, South Pacific, northern Australia, Indo-West Pacific, Red Sea.

Remarks. This species was collected under a coral rock. Dorsal side body color of is brown with irregular formed dark brown patches. Body size: $\mathrm{R}=3 \mathrm{~cm}, \mathrm{r}=0.7 \mathrm{~cm}, \mathrm{R}=4.3 \mathrm{r}$.

Family Goniasteridae Forbes, 1841

Genus Fromia Gray, 1840

\section{*11. Fromia nodosa A.M. Clark, 1967}

(Fig. 2D, 3C, F, M-O)

Fromia nodosa AM Clark, 1967b: 189; AM Clark and Rowe, 1971: 62; Mah, 2012: 213300.

Material examined. 1 specimen, Utwa diving 4, 11 Jan 2012. Distribution. Micronesia (Kosrae, Yap), Sri Lanka (Ceylon), West Indian Ocean (Amirante Island, Maldive Island), eastern Africa (Aldabra Atoll, Seychelles).

Remarks. This species was reported at the West Indian Ocean and eastern Africa (Clark, 1967b; Clark and Rowe, 1971) and this is the first reported from the Pacific Ocean. Body color is red, and the ventral part is white. Body size: $\mathrm{R}=2.6 \mathrm{~cm}, \mathrm{r}=0.7 \mathrm{~cm}, \mathrm{R}=3.7 \mathrm{r}$.

Family Oreasteridae Fisher, 1911

Genus Choriaster Lütken, 1869

\section{Choriaster granulatus Lütken, 1869}

Material examined. 2 specimens, Malem diving 1, 24 Jan 2011; 1 specimen, Tafunsak diving 2, 26 Jan 2011.

Distribution. Micronesia (Kosrae, Yap), southern Japan, southern China, Philippines, Guam, Palau, New Caledonia, Indo-West Pacific, eastern Africa, Red Sea.

Genus Culcita (L. Agassiz, 1836)

\section{Culcita novaeguineae Müller and Troschel, 1842}

Material examined. 1 specimen, Utwa diving 4, 11 Jan 2012.

Distribution. Micronesia (Kosrae, Yap), southern Japan, southern China, Philippines, Guam, Palau, Marshall Islands, Hawaii, northern Australia, Bengal Bay, Indo-West Paci- 
fic, eastern Africa (Madagascar, Mozambique).

Order Spinulosida Perrier, 1884

Family Echinasteridae Verrill, 1867

Genus Echinaster Müller and Troschel, 1840

*14. Echinaster luzonicus (Gray, 1840) (Fig. 4A-C)

Othilia luzonicus Gray, 1840: 282.

Echinaster luzonicus Müller and Troschel, 1842: 23; HL Clark, 1921: 98; 1946: 147; AM Clark and Rowe, 1971: 72; Rowe and Gates, 1995: 59; Mah, 2012: 367951.

Echinaster eridanella Müller and Troschel, 1842: 24; HL Clark, 1916: 62.

Material examined. 2 specimens, Malem diving 1, 24 Jan 2012; 1 specimen, Tafunsak diving 2, 26 Jan 2011.

Distribution. Micronesia (Kosrae, Yap), southern Japan, southern China, Philippines, Guam, Palau, South Pacific, northern Australia, New Caledonia.

Remarks. This species has five or six arms and rarely seven (Clark and Rowe, 1971). Our specimens have six arms. Body color of disk and basal side of arms is light orange and the terminal part of the arm is red.

Class Ophiuroidea Gray, 1840

Order Ophiurida Müller and Troschel, 1840

Infraorder Gnathophiurina Matsumoto, 1915

Family Ophiactidae Matsumoto, 1915

Genus Ophiactis Lütken, 1856

\section{*15. Ophiactis savignyi (Müller and Troschel, 1842)}

(Figs. 2D, 4D-F, I)

Ophiolepis savignyi Müller and Troschel, 1842: 95.

Ophiactis krebsii Lütken, 1856: 12; Ljungman, 1867: 323;

1872: 627; Duncan, 1879: 465.

Ophiolepis sexradia Grube, 1857: 343.

Ophiactis reinhardti Lütken, 1859, Pl. 3, fig. 7a, b.

Ophiactis sixradia: Lyman, 1865: 115; Koehler, 1898: 72.

Ophiactis savignyi: Ljungman, 1867: 323; HL Clark, 1915:

265; 1921: 108; Koehler, 1922: 164; Mortensen, 1933: 442;

AH Clark, 1939: 3; Mortensen, 1940: 70; HL Clark, 1946:

210; AH Clark, 1949: 31; AM Clark, 1967a: 47; AM Clark

and Rowe, 1971: 103; Pawson, 1978: 11; Shin and Rho,

1996: 411; Shin, 2012: 73; Stöhr and O'Hara, 2012: 125122.

Ophiactis maculosa von Martens, 1870: 248.

Ophiactis conferta Koehler, 1905: 25.

Ophiactis quadrispina HL Clark, 1915: 264.

Ophiactis versicolor HL Clark, 1939: 81.

Material examined. 11 specimens, Malem diving 1, 24 Jan 2011; 17 specimens, Tafunsak diving 1, 25 Jan 2011; 9 spe- cimens, Tafunsak diving 2, 26 Jan 2011; 2 specimens, Utwa diving 2, 9 Jan 2012; 1 specimen, Walung diving, 11 Jan 2012; 1 specimen, Utwa diving 5, 13 Jan 2012.

Distribution. Micronesia (Kosrae, Yap), Korea (Korea Strait, Jeju Island), Japan (Honshu, Kyushu), China, Philippines, Guam, northern Australia, Singapore, Indo-West Pacific, eastern Africa (Tanzania, Madagascar), Red Sea, Mediterranean Sea, Atlantic Ocean.

Remarks. This is a cosmopolitan species that usually inhabits the inner cavity of sponges. It was collected with sponges by SCUBA diving on Kosrae. Body color is light brown or greenish gray. Arms are usually six and rarely five.

Family Ophicomidae Ljungman, 1867

Genus Ophiocoma L. Agassiz, 1835

\section{Ophiocoma erinaceus Müller and Troschel, 1842} (Fig. 2E)

Material examined. 1 specimen, Utwa intertidal, 11 Jan 2012.

Distribution. Micronesia (Kosrae, Yap), southern Japan, southern China, Philippines, Guam, Hawaii, South Pacific, northern Australia, Indo-West Pacific, eastern Africa, Red Sea.

\section{Ophiocoma scolopendrina (Lamarck, 1816)} (Fig. 2F)

Material examined. 1 specimen, Lelu intertidal, 25 Jan 2011; 10 specimens, Tafunsak intertidal 1, 26 Jan 2011; 7 specimens, Tafunsak diving 2, 26 Jan 2011; 1 specimen, Tafunsak intertidal 2, 9 Jan 2012; 3 specimens, Utwa intertidal, 11 Jan 2012; 1 specimen, Tafunsak intertidal 3, 11 Jan 2012; 2 specimens, Walung intertidal, 12 Jan 2012.

Distribution. Micronesia (Kosrae, Yap), southern China, Guam, Saipan, Solomon Islands, Indo-West Pacific, eastern Africa (Mauritius, Madagascar), South Africa, Red Sea.

Genus Ophiomastix Müller and Troschel, 1842

\section{*18. Ophiomastix caryophyllata Lütken, 1869}

\section{(Fig. 4G, H, K, L)}

Ophiomastix caryophyllata Lütken, 1869: 43; HL Clark, 1946: 249; AM Clark and Rowe, 1971: 91; Lane et al., 2000: 482; Stöhr and O’Hara, 2012: 212391.

Material examined. 1 specimen, Tafunsak diving 2, 25 Jan 2011; 1 specimen, Utwa diving 2, 9 Jan 2012; 1 specimen, Utwa diving 3, 10 Jan 2012.

Distribution. Micronesia (Kosrae, Yap), southern Japan, 

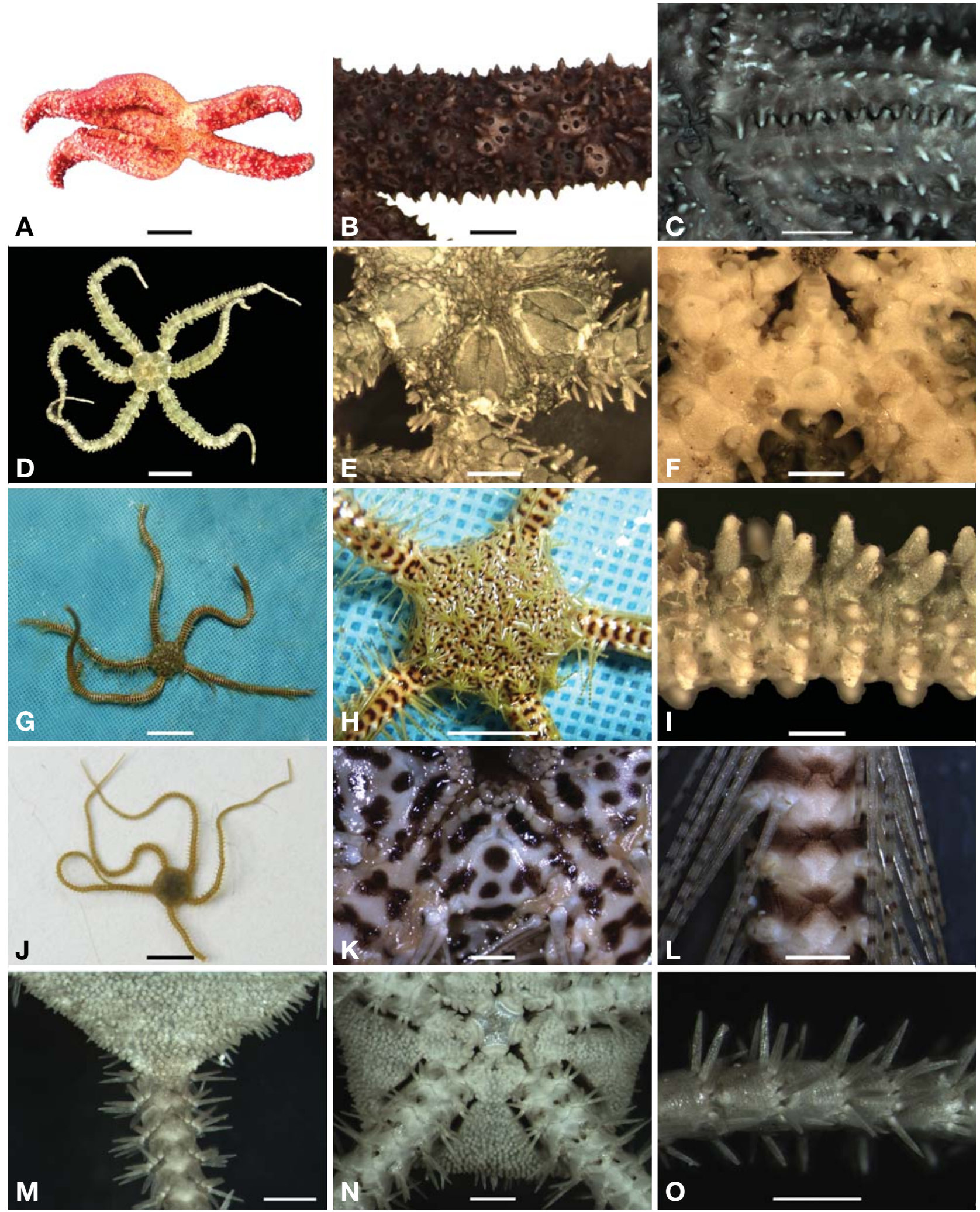

Fig. 4. Echinaster luzonicus $(\mathrm{A}-\mathrm{C})$, Ophiactis savignyi $(\mathrm{D}-\mathrm{F}, \mathrm{I})$, Ophiomastix caryophyllata $(\mathrm{G}, \mathrm{H}, \mathrm{K}, \mathrm{L})$, and Ophioconis pexmixta (J, M-O). A, D, G, J, Dorsal side; B, L, Dorsal side of arm; C, F, K, N, Oral part and ventral side of arm; $E, H$, M, Radial shields of disk and dorsal side arms; I, O, Arm spines. Scale bars: $A, H=1 \mathrm{~cm}, \mathrm{~B}, \mathrm{C}=2 \mathrm{~mm}, \mathrm{D}, \mathrm{J}=5 \mathrm{~mm}, \mathrm{E}, \mathrm{F}, \mathrm{I}, \mathrm{K}-\mathrm{M}=1 \mathrm{~mm}, \mathrm{G}=2 \mathrm{~cm}, \mathrm{~N}, \mathrm{O}=0.5 \mathrm{~mm}$. 
southern China, Guam, northeastern Australia, Indo-West Pacific, eastern Africa (Madagascar), Red Sea.

Remarks. These specimens were collected by SCUBA diving. Body is light cream color and has irregular formed black spots on the dorsal and ventral side of the disk. Arms are banded and are approximately equal width.

\section{Ophiomastix mixta Lütken, 1869}

Material examined. 2 specimens, Utwa diving 5, 13 Jan 2012.

Distribution. Micronesia (Kosrae), Korea (Jeju Island), southern Japan, southern China, Philippines, Guam, South Pacific, Australia, Indonesia (Ambon Island).

Remarks. This species is a common and abundant ophiuroid in the Indo-West Pacific, and at the subtidal zone of Jeju Island, Korea (Shin, 2012). Only two individuals were collected by SCUBA diving in Kosrae.

Family Ophiotrichidae Ljungman, 1867

Genus Ophiothrix Müller and Troschel, 1840

\section{Ophiothrix (Acanthophiothrix) purpurea von Martens, 1867}

Material examined. 3 specimens, Malem diving 1, 24 Jan 2011.

Distribution. Micronesia (Kosrae), southern China, Guam, Indo-West Pacific, eastern Africa (Madagascar, Tanzania), Red Sea.

Infraorder Ophiodermatina Smith, Paterson and Lafay, 1995 Family Ophiodermatidae Ljungman, 1867

Genus Ophioconis Lütken, 1869

\section{*21. Ophioconis permixta Koehler, 1905}

(Fig. 4J, M-O)

Ophioconis permixta Koehler, 1905: 14; AM Clark and Rowe, 1971: 127; Stöhr and O’Hara, 2012: 238160.

Material examined. 2 specimens, Utwa diving 3, 10 Jan 2012.

Distribution. Micronesia (Kosrae), Indonesia (Wowoni Island, Kai Island), East India Ocean, eastern Africa.

Remarks. Morphological characters of our specimens corresponded to the description of AM Clark and Rowe (1971). Body size: $\mathrm{R}=5 \mathrm{~mm}, \mathrm{r}=62 \mathrm{~mm}, \mathrm{R}=12.4 \mathrm{r}$.

Subphylum Echinozoa

Class Echinoidea Leske, 1778

Order Diadematoida Duncan, 1889
Family Diadematidae Gray, 1855

Genus Diadema Gray, 1825

\section{*22. Diadema savignyi (Audouin, 1829)}

Diadema savignyi: Michelin, 1845: 15; AM Clark and Rowe, 1971: 153; Rowe and Gates, 1995: 207; Kroh and Mooi, 2012: 213375.

Material examined. 1 specimen, Tafunsak intertidal 3, 11 Jan 2012.

Distribution. Micronesia (Kosrae, Yap), south Japan, south China, Philippines, Guam, South Pacific, northern Australia, Indo-West Pacific, West Indian Ocean, southeastern Arabia, eastern Africa (Kenya, Mauritius, Madagascar, Seychelles).

Remarks. This specimen was collected from the intertidal zone at night. Primary spines are very long and body color is black.

\section{Diadema setosum (Leske, 1778)}

Material examined. 2 specimens, Lelu intertidal, 25 Jan 2011; 1 specimen, Tafunsak diving 2, 26 Jan 2011; 1 specimen, Tafunsak diving 3, 27 Jan 2011; 1 specimen, Tafunsak intertidal 2, 9 Jan 2012; 1 specimen, Tafunsak intertidal 3, 11 Jan 2012.

Distribution. Micronesia (Kosrae), Korea (Jeju Island), Japan (Sagami bay-Bonin Islands), southern China, Taiwan, IndoWest Pacific.

Genus Echinothrix Peters, 1853

\section{*24. Echinothrix calamaris (Pallas, 1774)}

(Figs. 2G, 5A, D, G-I)

Echinus calamaris Pallas, 1774: 31, Pl. 2.

Echinothrix calamaris A Agassiz, 1873: 413; HL Clark, 1921: 146; Koehler, 1927: 47; HL Clark, 1932: 213; 1946: 300; AM Clark, 1967a: 51; Rowe and Gates, 1995: 208; Kroh and Mooi, 2012: 213377.

Echinothrix desorii A Agassiz, 1873: 451.

Material examined. 1 specimen, Tafunsak intertidal 3, 11 Jan 2012.

Distribution. Micronesia (Kosrae, Yap), southern Japan, southern China, Philippines, Hawaii, Solomon Islands, northern Australia, Indo-West Pacific, eastern Africa, Red Sea.

Remarks. The specimen, whose spines were moving smoothly, was collected from a wide horizontal coral rocky area of the intertidal zone at night. The primary spines on the dorsal side are white. Size is rather large and diameter of the test is about $9.7 \mathrm{~cm}$. 
A
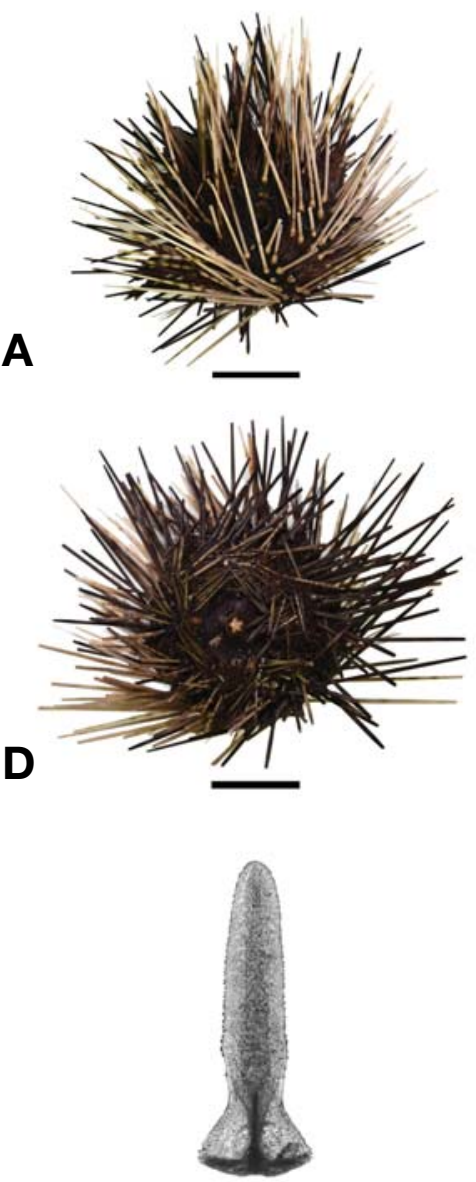

G
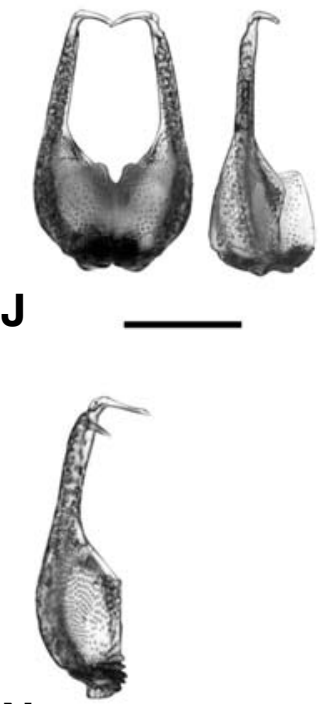

$\mathbf{N}$
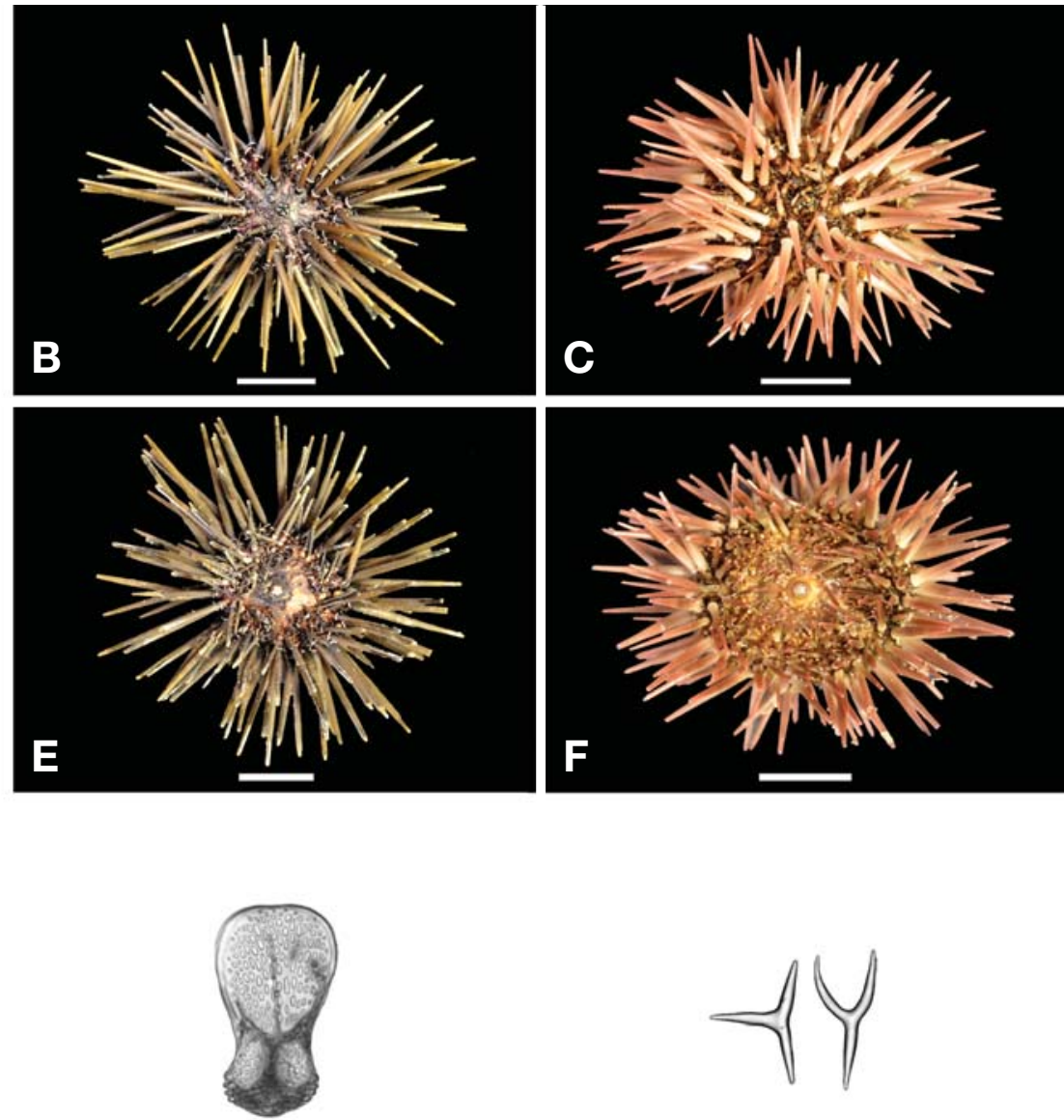

H
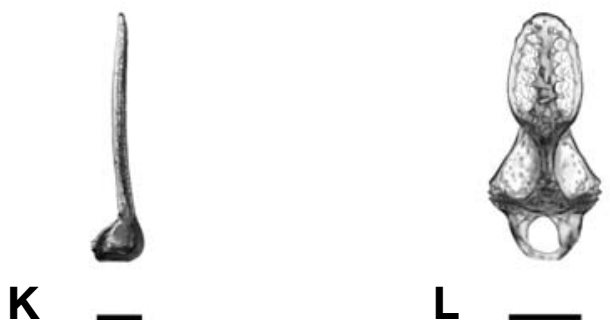

$\mathbf{L}$

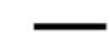

M
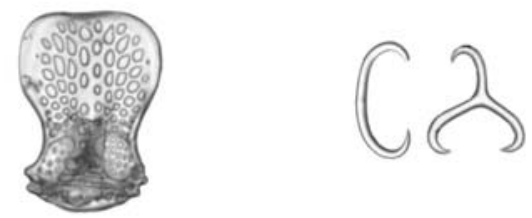

$\mathbf{P}$

Q

$\mathbf{R}$

Fig. 5. Echinothrix calamaris $(A, D, G-I)$, Paraselina gratiosa $(B, E, J-M)$, and Echinometra mathaei $(C, F, N-R)$. A-C, Dorsal side; $\mathrm{D}-\mathrm{F}$, Ventral side; G, K, O, Valve of tridentate pedicellaria; $\mathrm{H}, \mathrm{Q}$, Valve of tryphyllous pedicellaria; $\mathrm{I}, \mathrm{M}, \mathrm{R}$, Spicules of tubefeet; J, N, Valves of globiferous pedicellaria; $L, P$, Valve of ophiocephalous pedicellaria. Scale bars: $A, D=4.5 \mathrm{~cm}, B, C, E, F=2 \mathrm{~cm}, G, J, K=300$ $\mu \mathrm{m}, \mathrm{H}, \mathrm{L}, \mathrm{O}=100 \mu \mathrm{m}, \mathrm{I}, \mathrm{N}, \mathrm{P}-\mathrm{R}=50 \mu \mathrm{m}, \mathrm{M}=10 \mu \mathrm{m}$. 


\section{Echinothrix diadema (Linnaeus, 1758) (Fig. 2H)}

Material examined. 2 specimens, Lelu intertidal, 25 Jan 2011; 1 specimen, Tafunsak intertidal 2, 9 Jan 2012; 2 specimens, Malem intertidal, 10 Jan 2012; 4 specimens, Utwa intertidal, 11 Jan 2012; 2 specimens, Tafunsak intertidal 3, 11 Jan 2012; 1 specimen, Tafunsak intertidal 4, 13 Jan 2012.

Distribution. Micronesia (Kosrae, Yap), southern Japan, southern China, Philippines, Guam, Hawaii, South Pacific, northern Australia, Indo-West Pacific, eastern Africa, Red Sea.

Order Camarodonta Jackson, 1912

Family Parasaleniidae Mortensen, 1903

Genus Parasalenia A. Agassiz, 1863

\section{*26. Parasalenia gratiosa A. Agassiz, 1863}

(Figs. 2I, 5B, E, J-M)

Parasalenia gratiosa A Agassiz, 1863: 22; HL Clark, 1912: 369; 1925: 141; 1946: 331; Mortensen, 1948: 49; AM Clark and Rowe, 1971: 157; Kerr et al., 1992: 204; Rowe and Gates, 1995: 233; Kroh and Mooi, 2012: 212438.

Material examined. 1 specimen, Tafunsak diving 2, 26 Jan 2011; 1 specimen, Utwa diving 5, 13 Jan 2012.

Distribution. Micronesia (Kosrae), southern China, Guam, Indo-West Pacific, eastern Africa (Kenya, Madagascar, Mozambique), Red Sea.

Remarks. This species was collected at blue holes by SCUBA diving. Body color is nearly black but primary spine has white ring at its base.

Family Echinometridae Gray, 1855

Genus Echinostrephus A. Agassiz, 1863

\section{Echinostrephus aciculatus A. Agassiz, 1863}

Material examined. 4 specimens, Lelu intertidal, 25 Jan 2011; 1 specimen, Tafunsak intertidal 2, 9 Jan 2012.

Distribution. Micronesia (Kosrae, Yap), southern Japan, southern China, Taiwan, East Australia, Indo-West Pacific.

Genus Echinometra Gray, 1825

\section{*28. Echinometra mathaei (Blainville, 1825)}

(Figs. 2J, 5C, F, N-R)

Echinus mathai Blainville, 1825: 94.

Echinometra mathaei Blainville, 1834: 225; Mortensen, 1903: 128; A Agassiz and HL Clark, 1907: 241; HL Clark, 1908: 303; 1912: 372; Mortensen, 1940: 103; AM Clark and Rowe, 1971: 157; Rowe and Gates, 1995: 211; Kroh and
Mooi, 2012: 213383.

Echinometra heteropora L Agassiz and Desor, 1846: 372.

Echinometra microtuberculata A Agassiz, 1863: 22.

Echinometra picta A Agassiz and HL Clark, 1907: 241; HL Clark, 1912: 373.

Material examined. 1 specimen, Tafunsak intertidal 2, 9 Jan 2012; 1 specimen, Malem intertidal, 10 Jan 2012.

Remarks. This species is widely distributed in the Indo-West Pacific and South Pacific (Clark and Rowe, 1971; Rowe and Gates, 1995). Specimens of about $4 \mathrm{~cm}$ were collected under coral rocks at the intertidal zone. Tubefeet spicules are Cshaped and rarely a tridentate form with hooked tips.

Distribution. Micronesia (Kosrae, Yap), Korea (Jeju Island), southern Japan, southern China, Philippines, Guam, Solomon Islands, Hawaii, North Australia, Indo-West Pacific, Red Sea, eastern Africa.

Family Toxopneustidae Troschel, 1872

Genus Tripneustes L. Agassiz, 1841

\section{Tripneustes gratilla (Linnaeus, 1758) (Fig. 2K)}

Material examined. 1 specimen, Walung intertidal, 12 Jan 2012; 1 specimen, Tafunsak intertidal 4, 13 Jan 2012.

Distribution. Micronesia (Kosrae, Yap), Korea (Jeju Island), Japan (Sagami Bay, Boso Peninsula), southern China, Guam, Indo-West Pacific, southeastern Africa.

Order Clypeasteroida L. Agassiz, 1835

Family Clypeasteridae L. Agassiz, 1835

Genus Clypeaster Lamarck, 1801

\section{Clypeaster reticulatus (Linnaeus, 1758)}

Material examined. 3 specimens, Tafunsak intertidal 3, 11 Jan 2012

Distribution. Micronesia (Kosrae, Yap), southern Japan, southern China, Philippines, Hawaii, South Pacific, northern Australia, Indo-West Pacific, West Indian Ocean, eastern Africa.

Class Holothuroidea de Blainville, 1834

Order Apodida Brandt, 1835

Family Synaptidae Burmeister, 1837

Genus Euapta Östergren, 1898

\section{Euapta godeffroyi (Semper, 1868) (Fig. 2L)}

Material examined. 1 specimen, Lelu intertidal, 25 Jan 2011; 2 specimens, Utwa diving 3, 10 Jan 2012. 
Distribution. Micronesia (Kosrae), Guam, Indo-West Pacific, eastern Africa (Mauritius, Madagascar), Red Sea.

Genus Opheodesoma Fisher, 1907

*32. Opheodesoma clarki Heding, 1928 (Fig. 6D-K)

Opheodesoma clarki: AM Clark and Rowe, 1971: 184; Paulay, 2012: 241948.

Material examined. 1 specimen, Tafunsak intertidal 3, 11 Jan 2012.

Distribution. Micronesia (Kosrae), Philippines.

Remarks. Body color of dorsal side is dark reddish brown and ventral side is light brown.

Genus Synapta Eschscholtz, 1829

33. Synapta maculata (Chamisso and Eysenhardt, 1821)

Material examined. 1 specimen, Lelu intertidal, 25 Jan 2011; 3 specimens, Tafunsak intertidal 2, 9 Jan 2012; 3 specimens, Tafunsak intertidal 3, 11 Jan 2012; 4 specimens, Walung intertidal, 12 Jan 2012; 3 specimens, Tafunsak intertidal 4, 13 Jan 2012.

Distribution. Micronesia (Kosrae, Yap), Guam, Indo-West Pacific, eastern Africa (Kenya, Madagascar), Red Sea.

Order Aspidochirotida Grube, 1840

Family Holothuriidae Ludwig, 1894

Genus Actinopyga Bronn, 1860

34. Actinopyga mauritiana (Quoy and Gaimard, 1834)

Material examined. 2 specimens, Lelu intertidal, 25 Jan 2011; 1 specimen, Tafunsak intertidal 3, 11 Jan 2012.

Distribution. Micronesia (Kosrae), southern Japan, southern China, Philippines, Guam, Hawaii, Australia, Indo-West Pacific, eastern Africa (Mauritius), Red Sea.

\section{Actinopyga palauensis Panning, 1944}

Material examined. 3 specimens, Utwa intertidal, 11 Jan 2012; 1 specimen, Walung intertidal, 12 Jan 2012.

Distribution. Micronesia (Kosrae), Palau, New Caledonia.

Genus Bohadschia Jaeger, 1833

36. Bohadschia argus Jaeger, 1833 (Fig. 2M)

Material examined. 1 specimen, Tafunsak diving 2, 26 Jan
2011; 1 specimen, Walung diving, 11 Jan 2012.

Distribution. Micronesia (Kosrae), southern Japan, southern China, Philippines, Guam, South Pacific, northern Australia, Indonesia, Bengal Bay.

\section{Bohadschia bivittata (Mitsukuri, 1912)}

Material examined. 1 specimen, Tafunsak diving 3, 27 Jan 2011; 2 specimens, Tafunsak intertidal 3, 11 Jan 2012.

Distribution. Micronesia (Kosrae), Philippines, Caroline Islands, West Central Pacific.

\section{Bohadschia marmorata Jaeger, 1833}

Material examined. 2 specimens, Tafunsak intertidal 2, 9 Jan 2012; 1 specimen, Tafunsak intertidal 3, 11 Jan 2012.

Distribution. Micronesia (Kosrae, Yap), southern Japan, southern China, Philippines, South Pacific, northern Australia, Indo-West Pacific, Bengal Bay, eastern Africa, Red Sea.

Genus Holothuria Linnaeus, 1767

\section{Holothuria (Halodeima) atra Jaeger, 1833 (Fig. 2N)}

Material examined. 15 specimens, Lelu intertidal, 25 Jan 2011; 12 specimens, Tafunsak intertidal 1, 26 Jan 2011; 3 specimens, Malem intertidal, 10 Jan 2012; 1 specimen, Walung diving, 11 Jan 2012; 1 specimen, Walung intertidal, 12 Jan 2012; 4 specimens, Tafunsak intertidal 4, 13 Jan 2012.

Distribution. Micronesia (Kosrae), southern China, Guam, Indo-West Pacific, eastern Africa (Kenya, Mauritius, Madagascar, Mozambique), Red Sea.

\section{Holothuria (Halodeima) edulis Lesson, 1830} (Fig. 6A)

Material examined. 1 specimen, Tafunsak diving 1, 25 Jan 2011; 1 specimen, Tafunsak diving 3, 27 Jan 2011; 1 specimen, Walung diving, 11 Jan 2012.

Distribution. Micronesia (Kosrae), southern Japan, southern China, Philippines, Guam, South Pacific, northern Australia, Indonesia, Indo-West Pacific, Bengal Bay.

\section{Holothuria (Mertensiothuria) hilla Lesson, 1830}

Material examined. 1 specimen, Utwa diving 3, 10 Jan 2012; 1 specimen, Walung diving, 11 Jan 2012.

Distribution. Micronesia (Kosrae), Korea (Korea Strait, Jeju Island), southern Japan, Guam, Hawaii, Australia, Indo-West 


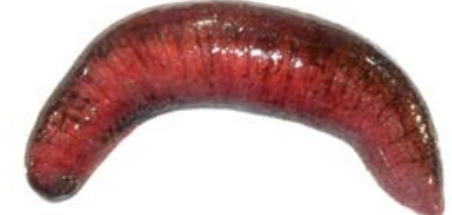

A
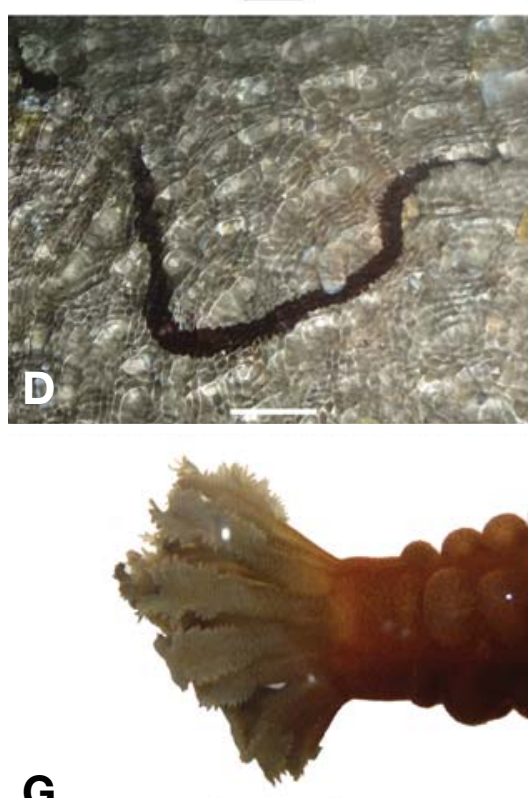

G
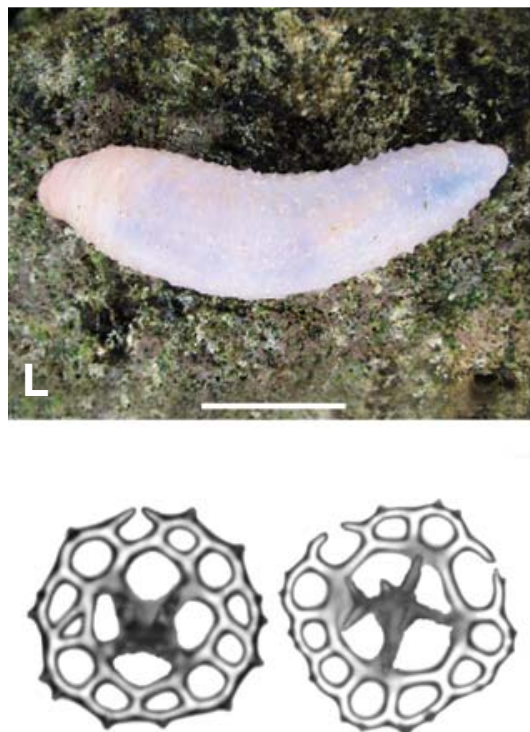

0

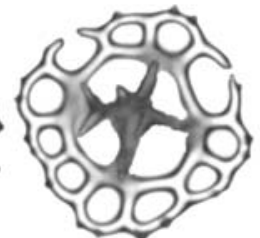

B
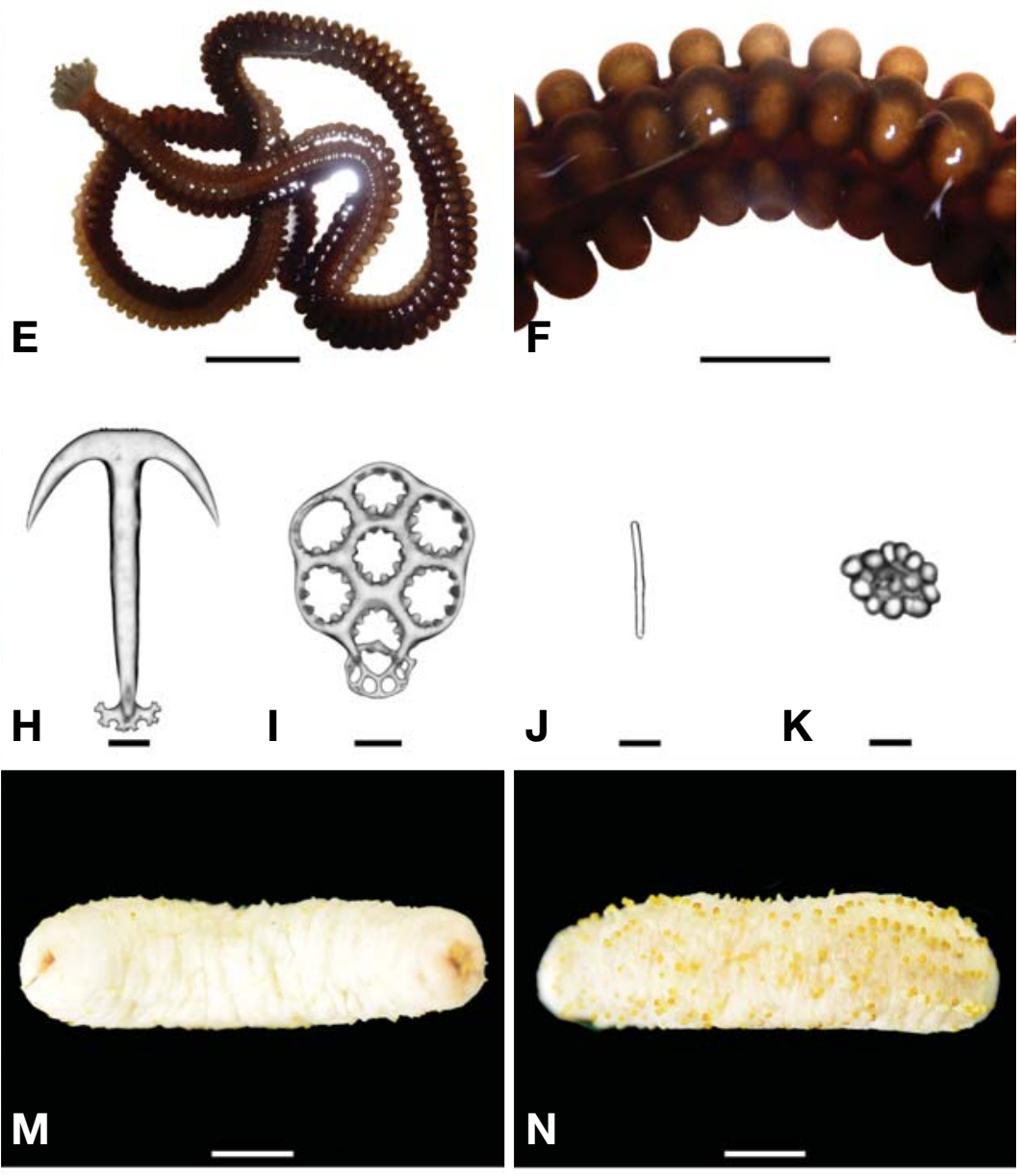

Fig. 6. Holothuria (Halodeima) edulis (A), H. (Mertensiothuria) leucospilota (B), Stichopus horrens (C), Opheodesoma clarki (D-K), and Labidodemas rugosum (L-Q). A-F, L, Living status; F, Body; G, Tentacles; $\mathrm{H}$, Anchor in body wall; I, Anchor plate in body wall; $\mathrm{J}$, Rod in tentacle; $\mathrm{K}$, Knobbed button in tentacle; $\mathrm{M}$, Dorsal side; $\mathrm{N}$, Ventral side; $\mathrm{O}$, Tables in body wall; $\mathrm{P}$, Lateral view of table in body wall; $Q$, Buttons in tubefeet. Scale bars: $A-C, F, G=2 \mathrm{~cm}, D=10 \mathrm{~cm}, E, L=5 \mathrm{~cm}, \mathrm{H}, \mathrm{I}, \mathrm{Q}=50 \mu \mathrm{m}, \mathrm{J}=20 \mu \mathrm{m}, \mathrm{K}=10 \mu \mathrm{m}, \mathrm{M}, \mathrm{N}=5$ $\mathrm{mm}, \mathrm{O}=0.5 \mathrm{~mm}, \mathrm{P}=250 \mu \mathrm{m}$. 
Pacific, Africa (Zanzibar).

\section{Holothuria (Mertensiothuria) leucospilota (Brandt, 1835) (Fig. 6B)}

Material examined. 2 specimens, Malem intertidal, 10 Jan 2012; 1 specimen, Utwa intertidal, 11 Jan 2012; 2 specimens, Tafunsak intertidal 3, 11 Jan 2012; 2 specimens, Walung intertidal, 12 Jan 2012; 1 specimen, Tafunsak intertidal 4, 13 Jan 2012.

Distribution. Micronesia (Kosrae), southern China, Guam, Indo-West Pacific, West Indian Ocean, eastern Africa (Madagascar), Red Sea.

\section{Holothuria (Stauropora) pervicax Selenka, 1867}

Material examined. 1 specimen, Lelu intertidal, 25 Jan 2011; 1 specimen, Tafunsak intertidal 2, 9 Jan 2012; 1 specimen, Utwa intertidal, 12 Jan 2012.

Distribution. Micronesia (Kosrae), southern China, Philippines, Guam, Hawaii, South Pacific, northern Australia, IndoWest Pacific, Indian Ocean, eastern Africa, Red Sea.

\section{Holothuria (Thymiosycia) impatiens (Forskål, 1775)}

Material examined. 1 specimen, Malem diving 2, 13 Jan 2012.

Distribution. Micronesia (Kosrae), Guam, Indo-West Pacific, West Indian Ocean, Africa (Kenya, Mauritius, Mozambique, Tanzania), Mediterranean Sea, Gulf of Mexico, Panama, Puerto Rico, Venezuela Sea.

Genus Labidodemas Selenka, 1867

*45. Labidodemas rugosum (Ludwig, 1875) (Fig. 6L-Q) Holothuria rugosa Ludwig, 1875: 110; HL Clark, 1946: 435; Domantay, 1954: 348.

Holothuria triremis Sluiter, 1901: 19.

Labidodemas rugosum: Rowe, 1969: 133; AM Clark and Rowe, 1971: 176; Sloan et al., 1979: 121; Cherbonnier, 1988: 53; Rowe and Gates, 1995: 304; Paulay, 2012: 210775.

Material examined. 1 specimen, Lelu intertidal, 25 Jan 2011. Remarks. This specimen was collected under coral rock. Body color is translucent white. Contracted body length in alcohol is $3 \mathrm{~cm}$ which is one-third of living body length. Distribution. Micronesia (Kosrae), southern China, Philippines, Guam, Malaysia, Indo-West Pacific, Indian Ocean, eastern Africa (Kenya, Madagascar, Somalia).
Genus Pearsonothuria Levin in Levin, Kalinin and Stonik, 1984

\section{Pearsonothuria graeffei (Semper, 1868)}

Material examined. 1 specimen, Malem diving 1, 24 Jan 2011.

Distribution. Micronesia (Kosrae, Yap), southern China, Philippines, Guam, Indo-West Pacific, Africa (Comoros, Madagascar), Red Sea.

Genus Stichopus Brandt, 1835

\section{Stichopus chloronotus Brandt, 1835 (Fig. 20)}

Material examined. 1 specimen, Tafunsak intertidal 3, 11 Jan 2012; 4 specimens, Walung intertidal, 12 Jan 2012.

Distribution. Micronesia (Kosrae, Yap), southern China, Guam, Indo-West Pacific, West Indian Ocean, eastern Africa (Comoros, Kenya, Madagascar, Mozambique), Red Sea.

\section{Stichopus herrmanni Semper, 1868}

Material examined. 2 specimens, Tafunsak diving 2, 26 Jan 2011.

Distribution. Micronesia (Kosrae), southern China, Philippines.

\section{Stichopus horrens Selenka, 1867 (Fig. 6C)}

Material examined. 1 specimen, Lelu intertidal, 25 Jan 2011; 1 specimen, Walung intertidal, 12 Jan 2012.

Distribution. Micronesia (Kosrae, Yap), southern Japan, southern China, Philippines, Hawaii, South Pacific, northern Australia, Indonesiam Indian Ocean (Maldive Island).

Order Dendrochirotida Grube, 1840

Family Sclerodactylidae Penning, 1902

Genus Afrocucumis Deichmann, 1944

\section{Afrocucumis africana (Semper, 1867)}

Material examined. 13 specimens, Lelu intertidal, 25 Jan 2011; 2 specimens, Malem intertidal, 10 Jan 2012; 2 specimens, Utwa intertidal, 11 Jan 2012; 2 specimens, Walung intertidal, 12 Jan 2012; 1 specimen, Tafunsak intertidal 4, 13 Jan 2012.

Distribution. Micronesia (Kosrae), Korea (Jeju Island), Japan (Kyushu-Ryukyu Islands), southern China, Guam, northern Australia, Malaysia, Indo-West Pacific, West Indian Ocean, eastern Africa (Madagascar). 


\section{DISCUSSION}

A faunistic study of echinoderms was performed at eight intertidal and 12 subtidal sites on Kosrae during January 24-28, 2011 and January 9-13, 2012 (Table 1, Fig. 1). In total, 276 specimens were collected and classified as 62 species: 50 species were identified based on morphological characteristics, and 12 were unidentified. Thirty-two echinoderm species, of which 28 species were identified, were collected in the intertidal zone. Thirty-five species were collected by SCUBA at the subtidal zone, and 27 were identified. The echinoderm fauna of the subtidal zone was more diverse than that of the intertidal zone. Seven echinoderms, including one asteroid (L. laevigata), one ophiuroid (O. savignyi), three echinoids (D. setosum, E. mathaei, and T. gratilla), and two holothuroids [A. africana and H. (M.) hilla] are distributed up to the Korea Strait and Jeju Island of Korea due to the warm Kuroshio Current. Holothuroids abundantly inhabited Kosrae, and $H$. (H.) atra, H. (M.) leucospilota, A. mauritiana, and S. chloronotus were particularly dominant species. $O$. scolopendrina was also a dominant ophiuroid and inhabited rock cracks at the wide rocky zone of station 5 (Utwa intertidal) (Table 1).

The echinoderm fauna of Kosrae was first reported by Kerr (1994) with regard to the Holothuroidea. Then, Kerr et al. (2008) surveyed 21 locations during 2 weeks (February 22March 7, 2008) and reported 74 species including 20 unidentified species. We newly reported 16 echinoderms from Kosrae which were not reported by Kerr (1994) or Kerr et al. (2008). In addition, some unidentified species need to be identified for further verification, including four crinoids (Comanthus sp., Lamprometra sp., Mariametridae sp.1 and sp.2), two asteroids (Aquilonastra sp. and Gomophia sp.), four ophiuroids (Ophiocoma sp., Ophiomastix sp., Ophionereis sp., and Ophiothrix sp.), and two holothuroids (Holothuria sp.1 and Holothuria sp.2).

\section{ACKNOWLEDGMENTS}

This study was supported by the Sahmyook University Research Fund, 2012 and a part of the project titled "Development of overseas marine biological resources and their utilization system" funded by the Ministry of Land, Transport and Maritime Affairs, Korea.

\section{REFERENCES}

Agassiz A, 1863. List of the echinoderms sent to different institutions in exchange for other specimens, with annotations. Bulletin of the Museum of Comparative Zoölogy at Harvard
College, 1:17-28.

Agassiz A, 1873. A revision of the echini. Part. 3. Memoirs of the Museum of Comparative Zoölogy at Harvard College, 3:383-628.

Agassiz A, Clark HL, 1907. Preliminary report on the echini collected, in 1902, among the Hawaiian Islands, by the U.S. Fish Commission steamer Albatross, in charge of commander Chauncey Thomas, U.S.N., commanding. Bulletin of the Museum of Comparative Zoology at Harvard College, 50: 231-259.

Agassiz L, Desor E, 1846. Catalogue raisonné des familles des genres et des espéces de la Classe de Echinoderms. Annals Science Naturelles, Zoologie, 3:325-374.

Blainville HM, 1825. Dictionnaire des sciences naturelles, dans lequel un traité méthodiquement des différences tetres de la nature, considérés soit en eux-memes, d'après l'état actuel de nos connoissances, soit relativement à l'utilité qu'en peuvent retierer la médicine, l'agriculture, le commerce et les arts. Vol. 37. Levrault, Paris, pp. 93-120.

Blainville HM, 1834. Manuel d'Actinologie ou de Zoophytologie. F.G. Levrault, Paris, pp. 1-644.

Cherbonnier G, 1988. Faune de Madagascar. Publiée sous les auspices du Gouvernement de la République Malgache, 70: 1-292.

Clark AH, 1908a. The crinoid genus Comatula Lamarck: with a note on the Encrinus parrae of Guerin. Proceeding of the United States National Museum, 33:683-688.

Clark AH, 1908b. The homologies of the arm joints and arm divisions in the recent crinoids of the families of the Comatulida and the Pentacrinitidae. Proceeding of the United States National Museum, 35:113-131.

Clark AH, 1909. On a collection of recent crinoids from the Philippine Islands. Proceeding of the United States National Museum, 36:391-410.

Clark AH, 1912. The crinoids of the Indian Ocean. Echinoderma of the Indian Museum, Part VII. Indian Museum, Calcutta, pp. 1-325.

Clark AH, 1921. A monograph of the existing crinoids, Vol. 1. The Comatulids, part 2. Bulletin of the United States National Museum, 82:1-795.

Clark AH, 1926. Notes on a collection of echinoderms from the Australian museum. Records of the Australian Museum, 15: 183-192.

Clark AH, 1939. Echinoderms (other than holothurians) collected on the presidential cruise of 1938. Smithsonian Miscellaneous Collections, 98:1-18.

Clark AH, 1949. Ophiuroidea of the Hawaiian Islands. Bulletin of the Bernice P. Bishop Museum, 195:1-133.

Clark AM, 1952. Some echinoderms from southern Africa. Transactions Royal Society of South Africa, 33:193-221.

Clark AM, 1967a. Echinoderms from the Red Sea, part 2 (crinoids, ophiuroids, echinoids and more asteroids). Israel South Read Sea Expedition 1962, Reports No. 21, 41:26-58.

Clark AM, 1967b. Notes on asteroids in the British Museum (Natural History). V. Nardoa and some other ophidiasterids. 
Bulletin of the British Museum (Natural History), 15:169198.

Clark AM, Rowe FWE, 1971. Monograph of shallow-water IndoWest Pacific echinoderms. Trustees of the British Museum (Natural History), 690:1-238.

Clark HL, 1908. Some Japanese and East Indian echinoderms. Bulletin of the Museum of Comparative Zoology at Harvard College, 51:279-311.

Clark HL, 1912. Hawaiian and other Pacific echini. Memoirs of the Museum of Comparative Zoölogy at Harvard College, 34:205-383.

Clark HL, 1915. Catalogue of recent ophiurans. Memoirs of the Museum Comparative Zoölogy at Harvard College, 25:163376.

Clark HL, 1916. Report on the sea-lilies, starfishes, brittle-stars and sea-urchins obtained by the F.I.S. Endeavour on the coasts of Queensland, New South Wales, Tasmania, Victoria, South Australia, and western Australia. Biological Results of the Fishing experiments carried on by the F.I.S. Endeavour 1909-1914, 4:1-123.

Clark HL, 1921. The echinoderm fauna of Torres Strait: its composition and its origin. Papers from the Department of Marine Biology of the Carnegie Institution of Washington, 10: 1-218.

Clark HL, 1925. A catalogue of the recent sea-urchins (Echinoidea) in the collection on the British Museum (Natural History). The British Museum Publication (Natural History), Department of Zoology, London, pp. 1-250.

Clark HL, 1932. Echinodermata (other than Asteroidea) of the Great Barrier reef expedition 1928-29. Scientific Reports of the Great Barrier Reef Expedition, 4:197-239.

Clark HL, 1939. Ophiuroidea. Scientific Reports of the John Murray Expedition, 6:29-136.

Clark HL, 1946. The echinoderm fauna of Australia: its composition and its origin. Carnegie Institution of Washington Publication, 566:1-567.

Domantay JS, 1954. Some holothurians from Guam and vicinity. Natural and Applied Science Bulletin, University of Philippines, 12:336-357.

Duncan PM, 1879. On some Ophiuroidea from the Korean Seas. Journal of the Linnean Society of London, Zoology, 14:445482.

Edward A, 2002. Marine biodiversity of the Federated States of Micronesia. Report of the Global Environmental Facility, FSM National Biodiversity Strategy and Action Plan Project. Department of Economic Affairs, Federated States of Micronesia, Palikir, pp. 1-20.

Fisher WK, 1913. New starfishes from the Philippine Islands, Celebes, and the Moluccas. Proceedings of the United States National Museum, 46:201-224.

Gray JE, 1840. A synopsis of the genera and species of the class Hypostoma (Asterias, Linnaeus). Annals of the Magazine of Natural History, 6:175-184, 275-290.

Grube AE, 1857. Diagnosen einiger neuer Echinodermen. Archiv für Naturgeschichte, 23:340-344.
Hartlaub C, 1890. Beitrag zur Kenntnis der Comatuliden fauna des Indischen Archipels. Nachrichten Von Der Gesellschaft Der Wissenschaften Zu Göttingen, 5:168-187.

Kerr AM, 1994. Shallow-water holothuroids (Echinodermata) of Kosrae, eastern Caroline islands. Pacific Science, 48:161174.

Kerr AM, Kim SW, Michonneau F, 2008. The shallow-water echinoderms of Kosrae. University of Guam Marine Laboratory Technical Report, 123:1-31.

Kerr AM, Netchy KH, Hoffman SM, 2007. The shallow-water echinoderms of Yap. University of Guam Marine Laboratory Technical Report, 121:1-34.

Kerr AM, Norris DR, Schupp PJ, Meyer KD, Pitlik TJ, Hooper DR, Chamberlain JD, Meyer LS, 1992. Range extensions of echinoderms (Asteroidea, Echinoidea and Holothuroidea) to Guam, Mariana Islands. Micronesica, 25:201-216.

Koehler R, 1898. Echinoderms recueillis par l'Investigator dans l'Ocean Indien, II les Opiures littorales. Bulletin Scientifique de la France et de la Belgique, 31:55-126.

Koehler R, 1905. Ophiures de l'expédition du Siboga. part 2. Ophiures littorales. Siboga Expeditie, 45:1-142.

Koehler R, 1922. Ophiurans of the Philippine Seas and adjacent waters. Smithsonian Institution United States National Museum Bulletin, 100:1-486.

Koehler R, 1927. Echinides du Musée Indien á Calcutta, III: Echinides réguliers. Echinoderma of the Indian Museum, 10:1-158.

Kroh A, Mooi R, 2012. World Echinoidea database [Internet]. The world register of marine species (WoRMS), Accessed 31 May 2012, <http://www.marinespecies.org/echinoidea>.

Lane DJW, Marsh LM, VandenSpiegel D, Rowe FWE, 2000. Echinoderm fauna of the South China Sea: an inventory and analysis of distribution patterns. The Raffles Bulletin of Zoology 2000 Supplement No. 8:459-493.

Ljungman AV, 1867. Ophiuroidea viventia huc usque cognita enumerat. Öfversigt af Kongliga Vetenskaps-Akademiens Förhandlingar, 24:303-336.

Ljungman AV, 1872. Förteckning öfver uti Vestindien af Dr. A. Goës samt under korvetten Josefinas expedition i Atlantiska Oceanen samlade Ophiurider. Öfversigt af Kungliga Vetenskapsakademiens Förhandlingar, 28:615-658.

Ludwig H, 1875. Beiträge zur Kenntniss der Holothurien. Arbeiten aus dem Zoologische Zootomischen Institut Würzburg, 2:77-120.

Lütken CF, 1856. Bidrag til kundskab om Slangestjernerne. II. Oversigt over de vestindiske Ophiurer. Videnskabelige Meddelelser fra Dansk Naturhistorisk Förening i Kjøbenhavn, 8:1-19.

Lütken CF, 1859. Additamenta ad historiam Ophiuridarum. Anden Afdelning. Det kongelige danske Videnskabernes Selskaps Skrifter. 5 Raekke, Naturvidenskabelig og mathematisk Afdelning, 5:177-271.

Lütken CF, 1869. Additamenta ad historiam Ophiuridarum. Tredie Afdelning. Det kongelige danske Videnskabernes Selskaps Skrifter. 5 Raekke, Naturvidenskabelig og mathema- 
tisk Afdelning, 8:20-109.

Lütken CF, 1871. Fortsatte kritiske og beskrivende Bidrag til Kundskab om Söstjernerne (Asteriderne). Videnskabelige Meddelelser fra Dansk Naturhistorisk Forening, 23:227-304.

Lyman T, 1865. Ophiuridae and Astrophytidae. Illustrated Catalogue of the Museum of Comparative Zoölogy at Harvard University, 1:1-200.

Mah C, 2012. World Asteroidea database [Internet]. The world register of marine species (WoRMS), Accessed 31 May 2012, <http://www.marinespecies.org/asteroidea>.

Messing CG, 2007. The crinoid fauna (Echinodermata: Crinoidea) of Palau. Pacific Science, 61:91-111.

Messing CG, 2012. Oxycomanthus bennetti, Phanogenia gracilis [Internet]. The world register of marine species (WoRMS), Accessed 31 May 2012, <http://www.marinespecies.org>.

Michelin H, 1845. Zoophytes, Echinodermes et Stellérides de l'Île Maurice. Magasin de Zoologie, 1845:1-27.

Mortensen T, 1903. The Danish Ingolf expedition 1895-1896. Vol. 4, No. 2. Echinoidea, part. 1. Bianco Luno, Copenhagen, pp. 1-198.

Mortensen T, 1933. Papers from Dr. Th. Mortensen's Pacific expedition 1914-16. 66. The echinoderms of St. Helena (other than crinoids). Videnskabelige Meddelelser fra Dansk naturhistorisk Forening, 93:401-473.

Mortensen T, 1940. Echinoderms from the Iranian Gulf. Asteroidea, Ophiuroidea, and Echinoidea. Danish Scientific Investigations in Iran, Part, 2:55-112.

Mortensen T, 1948. A monograph of the Echinoidea. IV (2). Clypeasteroida. Clypeasteridae, Arachnoidae, Fibulariidae, Laganidae and Scutellidae. C.A. Reitzel, Copenhagen, pp. $1-471$.

Müller J, 1841. Ueber die Gattungen und Arten der Comatulen. Verhandlungen der Königlichen Preussischen Akademie der Wissenschaften, Berlin, 6:179-189.

Müller J, Troschel FH, 1840. Beobachtungen über die Asteriensammlung des Zoologischen Museums. Monatsberichte der Königlichen Preussischen Akademie der Wissenschaften, Berlin, 1840:100-106.

Müller J, Troschel FH, 1842. System der Asteriden. Papier, Druck und Verlag, Braunschweig, pp. 1-134.

O'Loughlin PM, Rowe FWE, 2006. A systematic revision of the asterinid genus Aquilonastra O'Loughlin, 2004 (Echinodermata: Asteroidea). Memoirs of Museum Victoria, 63:257287.

O'Loughlin PM, Waters JM, 2004. A molecular and morphological revision of genera of Asterinidae (Echinodermata: Asteroidea). Memoirs of Museum Victoria, 61:1-40.
Pallas PS, 1774. Spicilegia Zoologica. Vol. 1. Fasc. 10. G. A. Lange, Berlin, pp. 1-44.

Paulay G, 2012. Labidodemas lugosum, Opheodesoma clarki [Internet]. The world register of marine species (WoRMS), Accessed 31 May 2012, <http://www.marinespecies.org>.

Pawson DL, 1978. The echinoderm fauna of Ascension Island, South Atlantic Ocean. Smithsonian Contributions to the Marine Sciences, 2:1-30.

Perrier E, 1869. Recherches sur les pédicellaires et les ambulacres des Astéries et des Oursins. Thèses présentées à la Faculté des Sciences de Paris pour obtenir le Grade de Docteur ès Sciences Naturelles Victor Masson et fils, Paris, pp. $1-188$.

Rowe FWE, 1969. A review of the family Holothuriidae (Holothurioidea: Aspidochirotida). Bulletin of the British Museum (Natural History) Zoology, 18:117-170.

Rowe FWE, Doty JE, 1977. The shallow-water holothurians of Guam. Micronesica, 13:217-250.

Rowe FWE, Gates J, 1995. Echinodermata. In: Zoological Catalogue of Australia. Vol. 33 (Ed., Wells A). CSIRO Australia, Melbourne, pp. 1-510.

Shin S, 2012. Brittle stars I: Echinodermata: Asterozoa, Ophiuroidea. In: Invertebrate fauna of Korea, Vol. 32 (Ed., National Institute of Biological Resources). National Institute of Biological Resources, Incheon, pp. 1-143.

Shin S, Rho BJ, 1996. Illustrated encyclopedia of fauna and flora of the Korea. Vol. 36. Echinodermata. Ministry of Education of Korea, Seoul, pp. 1-780.

Sloan NA, Clark AM, Taylor JD, 1979. The echinoderms of Aldabra and their habitats. Bulletin of the British Museum (Natural History) Zoology, 37:81-128.

Sluiter CP, 1901. Die Holothurien der Siboga expedition. Siboga expedite uitkomsten op Zoologische, Botanische, Oceanographische en Geologische gebeid verzameld in Nederland Oost-Indie 1899-1900 aan boord Hm. Siboga onder commano van Lt. ter zee 1e kl. GF. Tydeman uitgeven door Dr. M. Weber. Boekhandel en Drukkerij v. EJ. Brill, Leiden, Monograph, 44:1-142.

Stöhr S, O'Hara T, 2012. World Ophiuroidea database [Internet]. The world register of marine species (WoRMS), Accessed 31 May 2012, <http://www.marinespecies.org/ophiuroidea>.

Von Martens E, 1870. Die Ophiuriden des indischen Oceans. Archiv für Naturgeschichte, 36:244-262.

Received July 2, 2012 Revised December 30, 2012 Accepted January 10, 2013 